\title{
On the Nature of X\#Ray-Bright, Optically Normal Galaxies
}

\section{Citation}

Yuan, Feng, and Ramesh Narayan. 2004. "On the Nature of X\#Ray-Bright, Optically Normal Galaxies." The Astrophysical Journal 612 (2): 724-28. https://doi.org/10.1086/422802.

\section{Permanent link}

http://nrs.harvard.edu/urn-3:HUL.InstRepos:41384958

\section{Terms of Use}

This article was downloaded from Harvard University's DASH repository, and is made available under the terms and conditions applicable to Other Posted Material, as set forth at http:// nrs.harvard.edu/urn-3:HUL.InstRepos:dash.current.terms-of-use\#LAA

\section{Share Your Story}

The Harvard community has made this article openly available.

Please share how this access benefits you. Submit a story.

\section{Accessibility}




\title{
On the Nature of X-ray Bright Optically Normal Galaxies
}

\author{
Feng Yuan ${ }^{1,2}$ and Ramesh Narayan ${ }^{2}$
}

\begin{abstract}
Recent X-ray surveys by Chandra and XMM-Newton have revealed a population of X-ray bright, optically normal galaxies (XBONGs) at moderate redshifts. We propose that many XBONGs are powered by an inner radiatively inefficient accretion flow (RIAF) plus an outer radiatively efficient thin accretion disk. The absence of optical/UV activity in XBONGs is explained by the truncation of the thin disk near the black hole, while the relatively strong $\mathrm{X}$-ray emission is explained as inverse Compton emission from the hot RIAF. As an example, we show that the spectra of two XBONGs can be fit fairly well with such a model. By comparing these two sources to other accreting black holes, we argue that XBONGs are intermediate in their characteristics between distant luminous active galactic nuclei and nearby low-luminosity nuclei.
\end{abstract}

Subject headings: accretion, accretion disks — black hole physics — galaxies: active - galaxies: nuclei — hydrodynamics

\section{Introduction}

Recent deep X-ray surveys with ROSAT, Chandra and XMM-Newton have resolved more than $80 \%$ of the $0.1-10 \mathrm{keV}$ cosmic X-ray background into discrete sources (Hasinger et al. 1998; Mushotzky et al. 2000; Giacconi et al. 2001; Brandt et al. 2001). Optical identifications of these X-ray sources show that many are, as expected, luminous active galactic nuclei (AGNs). However, rather unexpectedly, a sizeable number of relatively bright X-ray sources have been spectroscopically identified with early-type "normal" galaxies without any obvious signature of nuclear activity in their optical spectra (Fiore et al. 2000; Hornschemeier et al. 2001; Giacconi et al. 2001; Barger et al. 2001; Comastri et al. 2002a,b).

\footnotetext{
${ }^{1}$ Department of Physics, Purdue University, West Lafayette, IN 47907; fyuan@physics.purdue.edu

${ }^{2}$ Harvard-Smithsonian Center for Astrophysics, 60 Garden Street, Cambridge, MA 02138; narayan@cfa.harvard.edu
} 
The existence of this unusual class of sources was already pointed out by Elvis et al. (1981) more than 20 years ago, based on an analysis of Einstein observations. It was later confirmed by Griffiths et al. (1995) using ROSAT data. The sources have been given a variety of names, such as Optically Dull Galaxies (Elvis et al. 1981), Passive Galaxies (Griffiths et al. 1995), X-ray Bright Optically Normal Galaxies (XBONGs, Comastri et al. 2002b), and Elusive Active Galactic Nuclei (Maiolino et al. 2003). We will adopt the name XBONG in this paper. According to Maiolino et al. (2003), the fraction of XBONGs among local galaxies is comparable to or even higher than that of optically selected Seyfert nuclei.

The large $\mathrm{X}$-ray-to-optical flux ratios of XBONGs, as well as their hard spectra in $\mathrm{X}$-rays (at least in the brighter sources for which spectral analysis is possible), suggest that AGN activity is occurring in these objects. The lack of evident optical emission lines is, however, a puzzle. There are several possible explanations.

One explanation is that XBONGs are luminous AGNs that happen to be heavily obscured. The obscuration must be in all directions, not just in a torus, since the sources lack both broad lines and narrow lines in their spectra (Marconi et al. 1994, 2000; Genzel et al. 1998; Spoon et al. 2000; Fabian 2003). Dudley \& Wynn-Williams (1997) predicted that a deep silicate absorption at $9.7 \mu \mathrm{m}$ should be detected in this case. The feature has been seen in two XBONGs (NGC 4945: Maiolino et al. 2000; NGC 4418: Spoon et al. 2001), indicating that the complete obscuration idea is correct for at least some XBONGs.

Despite this success, it seems unlikely that the obscuration model applies to all XBONGs. Severgnini et al. (2003) performed a detailed spectral analysis of three XBONGs observed with $X M M-N e w t o n$ and found that only one out of the three is X-ray obscured $\left(N_{H} \approx 2 \times 10^{23} \mathrm{~cm}^{-2}\right)$, while the other two sources are relatively unobscured $\left(N_{H} \approx\right.$ $4 \times 10^{21}, 1 \times 10^{21} \mathrm{~cm}^{-2}$, respectively). A similar result was obtained by Page et al. (2003), who carried out optical spectroscopy of a number of X-ray sources from the $13 \mathrm{hr} X M M$ Newton/Chandra deep survey. Of their 70 sources, 23 were found to be XBONGs, half of which were unabsorbed in X-rays.

A second explanation for XBONGs is that proposed by Moran, Filippenko, \& Chornock (2002) and Severgnini et al. (2003) according to which the lack of significant emission lines in XBONGs may be due to dilution of the nuclear spectrum by starlight from the host galaxy. However, the intrinsic optical continuum emission from XBONGs is weaker than in luminous, quasar-like AGNs (e.g., Comastri et al 2002b), which suggests that the emissionline luminosities are also intrinsically low. In terms of the spectral index $\alpha_{o x}$ (defined by $F_{\nu} \propto \nu^{-\alpha_{\mathrm{ox}}}$ ) between $2500 \AA$ and $2 \mathrm{keV}$, Severgnini et al. (2003) find that $\alpha_{o x} \approx 1.2$ for a small sample of XBONGs. This value is systematically smaller than $\alpha_{o x} \approx 1.5$ for luminous AGNs (Brandt, Laor \& Wills 2000). 
A third possibility is that XBONGs are BL Lac-like objects. Observations by Chandra and XMM-Newton of at least one XBONG, the source "P3" (§2.2), indicate no significant flux or spectral variability over a time interval of seven months (Comastri et al. 2002a). This is highly unusual for a BL Lac object. The presence of a large calcium break and a lack of detectable radio emission in several XBONGs (Fiore et al. 2000) are additional arguments against the BL Lac model. Nevertheless, this model is hard to rule out and may well describe some XBONGs, though it seems unlikely to apply to a majority of XBONGs.

The last possibility, the one we focus on in this paper, is that the intrinsic weakness of XBONGs in the optical/UV waveband is because these sources lack an optically-thick accretion disk at small radii. Instead of a cool disk, we suggest that the gas at small radii is in the form of a very hot radiatively inefficient accretion flow (RIAF, also known as an advection-dominated accretion flow or ADAF). We discuss this model in $\S 2$ and show that it is qualitatively consistent with the spectra of two XBONGs $(\S \S 2.1,2.2)$. We conclude in $\S 3$ with a summary and discussion.

\section{RIAF Model of XBONGs}

The redshift and luminosity distribution of XBONGs provide clues to the nature of these sources. In a sample of 71+45 hard X-ray sources selected from the HELLAS2XMM and Chandra surveys, Comastri et al. (2002b) found 10 XBONGs. The 10 sources are at low redshift and have low luminosities. Brusa et al. (2003) have presented optical identifications for a sample of 35 sources, selected again from the HELLAS2XMM survey. They find that at low redshift, the sources consist of a mix of Broad Line AGNs, Narrow Emission-Line galaxies and XBONGs, whereas at $z>1$ all the sources are spectroscopically classified as Broad Line AGNs. Similarly, the 23 XBONGs identified by Page et al. (2003) all lie at $z<1$ and have lower X-ray luminosities, whereas the non-XBONG sources in their sample extend over a wide range of redshift and are typically brighter.

These results indicate that XBONGs have lower luminosities compared to standard AGNs. The fact that the sources are also found preferentially at lower redshift follows naturally. Supermassive black holes in the nuclei of high redshift galaxies are generally believed to have a lot of gas available for accretion and are therefore quite luminous. At lower redshifts there should be less gas and we expect the nuclei to be less luminous. Therefore, if XBONG behavior is associated with lower luminosities, then the sources should be found at somewhat lower redshifts than bright AGNs. But why should lower luminosity AGNs have proportionately less optical emission compared to more luminous objects? We propose that these sources do not have a standard geometrically-thin optically-thick disk at small radii. 
Instead, the disk is truncated at a "transition" radius $R_{\mathrm{tr}}$, and the accretion flow inside $R_{\mathrm{tr}}$ is in the form of an optically-thin RIAF. (Early versions of the RIAF model were called ADAFs, Narayan \& Yi 1995; see Narayan, Mahadevan \& Quataert 1998 and Kato, Fukue, \& Mineshige 1998 for reviews). Because of the absence of an optically-thick disk at small radii, the source emits much less optical and UV radiation.

This paradigm - an inner RIAF plus an outer standard thin disk - has been shown to work very well (even required) in several types of sources: black hole X-ray binaries in the hard state (Esin, McClintock \& Narayan 1997; Poutanen, Krolik \& Ryde 1997; Dove et al. 1998; see McClintock \& Remillard 2003 and Zdziarski \& Gierlinski 2004 for reviews), lowluminosity AGNs in our local universe (Quataert et al. 1999; Shields et al. 2000), and some Seyfert 1 galaxies (Chiang 2002; Chiang \& Blaes 2003). Quataert et al. (1999) estimated $R_{\mathrm{tr}} \approx 100 R_{s}$ for the LLAGNs, M 81 and NGC 4579, while Chiang \& Blaes (2003) estimated $R_{\mathrm{tr}} \approx 30-80 R_{s}$ for the Seyfert 1 galaxy NGC 5548. The lack of a big blue bump in the spectra of LLAGNs (Ho 1999) indicates that an optically-thick disk is not present at small radii in these sources, and there is a similar suggestion in the case of NGC 5548 from the lack of a relativistically broadened and skewed Fe $\mathrm{K} \alpha$ line in its X-ray spectrum (Pounds et al. 2003). As in the case of the XBONGs, all these sources have relatively low optical luminosities compared to quasars. M81 and NGC 4579 have bolometric luminosities $\approx 10^{-4}$ Eddington, while NGC 5548 has a luminosity of $\approx 10^{-2}$ Eddington. Finally, as mentioned in $\S 1$, Severgnini et al. (2003) measured a spectral index $\alpha_{\text {ox }} \approx 1.2$ for three XBONGs, which is lower than $\alpha_{\mathrm{ox}} \approx 1.5$ for typical AGNs. In comparison, LLAGNs have $\alpha_{\mathrm{ox}} \approx 0.75-1.08$, with a mean value of 0.9 (Ho 1999), and NGC 5548 has $\alpha_{\text {ox }} \approx 1.1$ (Chiang \& Blaes 2003).

In view of all these indications that XBONGs are spectrally similar to LLAGNs and some Seyfert 1 galaxies, it is reasonable to suppose that the same accretion geometry, viz., an inner RIAF plus an outer thin disk, is present in all the sources. Comastri et al. (2002a) have ruled against such a model for XBONGs using two different arguments. First, they used the variability trend found by Nandra et al. (1997) to argue that if XBONGs are lowluminosity AGNs, they should be highly variable, whereas there is no evidence for significant variability. However, Ptak et al. (1998) have shown that AGNs with luminosities below a certain threshold do not follow the Nandra et al. trend. Indeed, Ptak et al. cite this as evidence that LLAGNs have ADAFs/RIAFs. Second, Comastri et al. (2002a) compared the spectrum of the XBONG source P3 with certain model spectra from Quataert \& Narayan (1999) and claimed that the radio flux of the source is incompatible with a RIAF model. However, such a comparison is very crude; for example, the effect of the mass of the black hole was not taken into account. In fact, the detailed calculations presented below show that a RIAF model is consistent with the data. 
We now analyze in detail two XBONG sources and show that the model explains the main features of their spectral energy distributions.

\subsection{Source \#1}

The spectral data available on XBONGs is generally rather limited, with XMMJ021822.3050615.7, or "Source \#1" (Severgnini et al. 2003), having perhaps the best data (see Figure 1). A radio flux is available from $V L A$ observations, and X-ray data have been obtained with XMM-Newton. The X-ray luminosity is $L_{2-10 \mathrm{keV}}=5.6 \times 10^{42} \mathrm{erg} \mathrm{s}^{-1}$, and the X-ray photon index is $\Gamma=1.66 \pm 0.30$. Severgnini et al. (2003) measured $\alpha_{o x}=1.2 \pm 0.1$, which gives the $2500 \AA$ data point shown in Figure 1. Using the Magorrian et al. (1998) relation between black hole mass and bulge luminosity, we estimate the black hole mass to be $M_{\mathrm{bh}} \approx 3 \times 10^{9} M_{\odot}$. This value should be regarded as an upper limit (Ferrarese \& Merritt 2000).

We have modeled the spectrum of Source \#1 using a current version of the RIAF model. Recent numerical simulations (Stone, Pringle, \& Begelman 1999; Hawley \& Balbus 2002; Igumenshchev et al. 2003) and analytical work (Blandford \& Begelman 1999; Narayan et al. 2000; Quataert \& Gruzinov 2000) indicate that probably only a fraction of the gas that is available at large radius in a RIAF actually accretes onto the black hole. The rest of the gas is either ejected from the flow or is prevented from accreting by convective motions. However, the details are likely to depend on the accretion rate. In the case of XBONGs, since the accretion rate is probably moderately high, the Bernoulli parameter as well as the entropy gradient are likely to approach zero. As a result, outflows and convection may be less well-developed. Given the theoretical uncertainty, we consider two different kinds of models. In the first model, we assume that the accretion rate in the RIAF varies with radius as

$$
\dot{M}_{\mathrm{RIAF}}(R)=\dot{M}_{0}\left(\frac{R}{R_{\mathrm{tr}}}\right)^{s}, \quad R \leq R_{\mathrm{tr}} .
$$

Here $R_{\mathrm{tr}}$ is the transition radius between the outer thin disk and the inner RIAF, and $\dot{M}_{0}$ is the accretion rate of the RIAF at $R_{\mathrm{tr}}$. We assume $s=0.3$ as in the case of Sgr A* (Yuan, Quataert \& Narayan 2003), and we correspondingly set $\delta$, the fraction of the turbulent energy that heats the electrons, equal to 0.5 (see Quataert \& Gruzinov 1999). In the second model, we assume that the accretion rate is independent of radius, i.e., $s=0$, and we correspondingly set $\delta=10^{-2}$ (as in the original ADAF model, e.g., Narayan et al. 1998). We solve the radiation-hydrodynamic equations of the RIAF self-consistently to obtain the profiles of density and electron temperature, and we then calculate the emergent spectrum (see Yuan, Quataert \& Narayan 2003 for details). The radiative processes we consider in the 
RIAF include synchrotron and bremsstrahlung emission, and the Comptonization of these photons as well as soft photons from the outer thin disk. For the thin disk, we assume that the emitted spectrum is locally a blackbody. We include both the energy from viscous dissipation within the disk and the reprocessing of hot radiation impinging from the RIAF.

The thick solid line in Figure 1 shows the predicted spectrum of the first model (with $s=0.3, \delta=0.5)$ for $\dot{M}_{0}=10^{-2} \dot{M}_{\text {Edd }}$ and $R_{\text {tr }}=60 R_{s}$. The dashed line shows the spectrum from the RIAF alone and the dot-dashed line from the outer thin disk alone. The combined spectrum fits the optical and X-ray data quite well. The under-prediction of the radio data is common in RIAF models (e.g., Quataert et al. 1999). The flux would be enhanced if the source has a jet (e.g., Yuan, Markoff, \& Falcke 2002a; Yuan et al. 2002b), or if the RIAF has nonthermal electrons (e.g., Mahadevan 1999; Özel, Psaltis \& Narayan 2000; Yuan, Quataert \& Narayan 2003). We have not taken these effects into account in the present paper. The transition radius in the model is a little smaller than in the models of M81 and NGC 4579 $\left(\approx 100 R_{s}\right.$; Quataert et al. 1999), but appear to be similar to that found for the Seyfert 1 galaxy NGC $5548\left(\approx 30-80 R_{s}\right.$; Chiang \& Blaes 2003$)$. The smaller radius explains why $\alpha_{\text {ox }}$ is larger in Source \#1 and NGC 5548 compared to LLAGNs. The thin solid line in Figure 1 shows the result of the second model (with $s=0, \delta=10^{-2}$ ). Because of a degeneracy between $s$ and $\delta$ (see Quataert \& Narayan 1999), the parameters are very similar to the thick solid line, $\dot{M}=10^{-2} \dot{M}_{\text {Edd }}$ and $R_{\mathrm{tr}}=40 R_{s}$.

The low optical flux of the model is a direct consequence of truncating the thin disk at $R_{\mathrm{tr}}$. For comparison, we show by the three dotted lines the emission from three standard thin disks extending down to the innermost stable circular orbit (ISCO) at $3 R_{S}$, with accretion rates of $\dot{M}_{0}=10^{-3}, 2 \times 10^{-4}$, and $5 \times 10^{-5} \dot{M}_{\text {Edd }}$, respectively. The upper two models predict too much flux in the optical and are immediately ruled out. Although the model with $\dot{M}_{0}=5 \times 10^{-5} \dot{M}_{\text {Edd }}$ can reproduce the $2500 \AA$ flux, it would require the X-ray flux (which is presumably produced by a corona above the disk) to be $\approx 50 \%$ of the bolometric luminosity, which is highly unusual for an AGN.

In the above models, the mass of the black hole we have adopted is an upper limit. It is not easy to estimate the uncertainty in the mass, but fortunately this parameter does not affect any of the conclusions. For instance, if the black hole mass is 10 times lower, i.e., $M_{\mathrm{bh}} \approx 3 \times 10^{8} M_{\odot}$, we only need to change the parameters to $\dot{M}_{0} \approx 2 \times 10^{-2} \dot{M}_{\mathrm{Edd}}$ and $R_{\mathrm{tr}} \approx 55 R_{s}$, while keeping the other parameters unchanged $(s=0.3, \delta=0.5)$, to match the data. Also, we find that a standard thin disk extending down to the ISCO is still inconsistent with the data. 


\subsection{Source P3}

The second object we analyze is the source P3, which is the first known example of an XBONG (Fiore et al. 2000). This source is interesting because Comastri et al. (2002a) claimed that the RIAF model cannot fit its spectrum. The mass of the central black hole is estimated from the observed $\mathrm{B}$ luminosity of the galaxy bulge to be $M_{\mathrm{bh}} \approx 4 \times 10^{8} M_{\odot}$ (Fiore et al. 2000). As before, this is an upper limit on the mass.

Multiwavelength observations of P3 were carried out by Comastri et al. (2002a) and the results are plotted in Figure 2. Radio observations at $5 \mathrm{GHz}$ give a $3 \sigma$ upper limit of $0.15 \mathrm{mJy}$. Near-infrared and optical observations again give only upper limits which are not very useful to constrain models. We show the optical upper limits in Figure $2 .{ }^{1}$ In addition, we arbitrarily assume that the value of the spectral index $\alpha_{\text {ox }}$ is identical to that of Source $\# 1$, i.e., $\alpha_{\mathrm{ox}}=1.2$. The approximate optical luminosity at $2500 \AA$ thus calculated is indicated by the filled circle in the optical band. P3 was observed in X-rays by both Chandra and XMM-Newton. Unfortunately, due to the weakness of the source, only a small number of counts were obtained, $\approx 60$ and 200 in the two observations. The Chandra data give a photon index of $\Gamma \approx 1.4$, assuming a Galactic column density. For the XMM-Newton observations, acceptable fits are obtained with a power-law of photon index $\Gamma=1.1 \pm 0.35$, or $\Gamma=1.8$ (an average value for AGNs). The latter fitting gives an absorption-corrected $2-10 \mathrm{keV}$ luminosity of about $3 \times 10^{42} \mathrm{erg} \mathrm{s}^{-1}$. The X-ray "bow-tie" corresponds to the two extremes of the $\mathrm{X}$-ray data, with two photon indices $\Gamma=1.1$ and 1.8 .

Comastri et al. (2002a) considered the obscuration model in detail for P3. Assuming an average value of the optical-to-X-ray flux ratio typical of hard X-ray-selected unobscured quasars, and taking a standard Galactic extinction curve, they calculated the expected optical magnitude of the source corresponding to the best-fit $N_{\mathrm{H}}$ from the X-ray analysis. They concluded that the nuclear emission would probably have been detected in the optical band, in conflict with observations. In fact, even when they assumed that the obscuring material is Compton-thick $\left(N_{H} \gtrsim 1.5 \times 10^{24} \mathrm{~cm}^{-2}\right.$ for which there is no observational evidence), by comparing the spectra of P3 and NGC 6240, the prototype of a heavily obscured AGN, they found that although they could explain the absence of optical activity in P3, the radio and IR flux of the source are significantly lower than that of NGC 6240.

The thick solid line in Figure 2 shows the spectrum predicted by a RIAF model of P3 with $\dot{M}_{0}=1.3 \times 10^{-2} \dot{M}_{\mathrm{Edd}}, R_{\mathrm{tr}}=60 R_{s}$. These parameters are similar to those of Source

\footnotetext{
${ }^{1}$ Note that an extinction-correction has been applied to the optical limits using the $N_{\mathrm{H}}$ value derived from XMM-Newton data
} 
\#1. The dashed line shows the spectrum from the RIAF alone and the dot-dashed line from the outer thin disk alone. The combined spectrum satisfies all the available constraints. In particular, we see that the model is consistent with the upper limit on the radio flux, contrary to the claim of Comastri et al. (2002a). The three dotted lines show the emission from three standard thin disks extending down to the ISCO, with accretion rates of $\dot{M}_{0}=$ $8 \times 10^{-5}, 8 \times 10^{-4}$, and $10^{-2} \dot{M}_{\text {Edd }}$, respectively. As in the case of Source $\# 1$, none of these models fits the spectrum well.

\section{Summary and Discussion}

We have investigated in this paper the nature of the population of X-ray bright, optically normal galaxies, or XBONGs. While the absence of optical activity in some XBONGs may be due to obscuration, this explanation is unlikely to apply to all XBONGs. We consider the possibility that many XBONGs possess radiatively inefficient accretion flows (RIAFs, formerly ADAFs). According to this model, the accretion flow occurs as a geometricallythin optically-thick disk for radii larger than a transition radius $R_{\mathrm{tr}}$, and as an optically-thin RIAF for radii below $R_{\mathrm{tr}}$. Because of the absence of an optically-thick disk at small radii, there is very little optical or UV emission, and therefore very little broad or narrow line emission. Since RIAFs are possible only at small accretion rates (Narayan \& Yi 1995; Esin et al. 1997), XBONGs are expected to populate the low-redshift, low-luminosity end of the AGN distribution. This is confirmed by observations.

XBONGs are spectrally very similar to LLAGNs in our local universe as well as some Seyfert 1 galaxies. All of these sources have weak optical/UV emission and relatively large $\mathrm{X}$-ray fluxes. The spectral index $\alpha_{o x}$ between $2500 \AA$ and $2 \mathrm{keV}$ is $\approx 1.2$ for XBONGs, $\approx 1.1$ for the Seyfert 1 galaxy NGC 5548, and $\approx 0.9$ for a small sample of LLAGNs. These values are small compared to $\alpha_{o x} \approx 1.5$ for luminous AGNs. Both LLAGNs and NGC 5548 have been successfully modeled by RIAFs (Quataert et al. 1999; Chiang \& Blaes 2003). The case for considering a similar model for XBONGs is thus strong. To test this proposal, we have modeled two XBONGs, Source \#1 (§2.1) and P3 (§2.2). We find that the RIAF model fits the available spectral data on both sources reasonably well (Figures 1,2). We also find that any model in which the thin disk comes all the way down to the innermost stable circular orbit (ISCO) is inconsistent with the data.

It is useful to place XBONGs in the context of other accreting black hole sources. Figure 3 shows the transition radius $R_{\mathrm{tr}}$ in Schwarzschild units and the luminosity $L$ in Eddington units for a number of objects whose spectra have been fitted with the RIAF model. Luminous black hole sources, including AGNs at high redshifts and black hole X-ray binaries in the high 
soft state, have standard radiatively efficient accretion disks extending down to the ISCO. When the luminosity is below a certain value, say $\approx 0.03 L_{\mathrm{Edd}}$, an advection-dominated RIAF is allowed (Esin et al. 1998), and it is postulated that the thin disk is then truncated and the innermost region of the flow is replaced by a RIAF. It is also expected that the lower the luminosity $L / L_{\mathrm{Edd}}$ the larger the transition radius $R_{\mathrm{tr}} / R_{s}$ (e.g., Narayan \& Yi 1995; Esin et al. 1997; Narayan et al. 1998; Rózańska \& Czerny 2000; Manmoto \& Kato 2000). Such a correlation is clearly seen in Figure 3 . In order of decreasing luminosity $L / L_{\text {Edd }}$, the sources shown are: 1) X-ray binaries in the soft state and bright AGNs; 2) X-ray binaries in the hard state and Seyfert 1 galaxies; 3) XBONGs; 4) LLAGNs; 5) X-ray binaries in the quiescent state; 6) ultra-dim AGNs. Note that we have analyzed only two XBONGs in this paper, both of which appear to be brighter than typical LLAGN when measured in Eddington units. As a class, however, XBONGs are quite heterogeneous, and it is possible that other XBONGs are similar in luminosity to LLAGNs, or perhaps even dimmer.

One caveat to note is that our estimates of $R_{\mathrm{tr}} / R_{S}$ for Source \#1 and P3 are sensitive to the assumed optical fluxes of the two sources. The optical flux of Source \#1 was obtained by Severgnini et al. (2003) by an indirect method, while P3 has no optical measurement and we have arbitrarily assumed the same flux as in Source \#1. Our estimates of the transition radii are thus rather uncertain.

Finally, while we have suggested in this paper that a RIAF is present in many XBONGs, we do not mean to imply that the RIAF necessarily produces the entire emission. It is possible, for instance, that some of the radiation (e.g., radio) comes from a jet. The BL Lac-type model mentioned in $\S 1$ is an extreme example of this scenario in which most of the emission comes from a highly relativistic and beamed flow. Another significant zone of emission is the base of the jet, or the region where the jet and the disk meet (e.g., see Livio, Pringle \& King 2003). The Doppler factor here is expected to be small so variability should be weak. A model of this region has been successfully worked out for Sgr A* (Yuan, Markoff, \& Falcke 2002a) and NGC 4258 (Yuan et al. 2002b).

We thank our second referee for a number of useful comments which helped improve the manuscript greatly. This work was supported in part by NSF grant AST-0307433 and NASA grants NAG5-9998 and NAG5-10780. 


\section{REFERENCES}

Barger, A.J., Cowie, L.L., Mushotzky, R.F., \& Richards, E.A., 2001, AJ, 121, 662

Blandford, R.D., \& Begelman, M.C. 1999, MNRAS, 303, L1

Brandt, W.N., Alexander, D.M., Hornschemeier, A.E. et al. 2001, AJ, 122, 2810

Brandt, W.N., Laor, A., \& Wills, B.J. 2000, ApJ, 528, 637

Brusa, M., Comastri, A., Mignoli, M., Fiore, F., et al. 2003, A\&A, 409, 65

Chiang, J. 2002, ApJ, 572, 79

Chiang, J., \& Blaes, O. 2003, ApJ, 586, 97

Comastri, A., Mignoli, M., Ciliegi, P., et al., 2002a, ApJ, 571, 771

Comastri, A. et al. 2002b, astro-ph/0203019

Di Matteo, T., Allen, S.W., Fabian, A.C., Wilson, A.S., Young, A.J., 2003, ApJ, 582, 133

Dove, J. B., Wilms, J., Nowak, M. A., Vaughn, B. A., \& Begelman, M. C. 1998, MNRAS, 292,729

Dudley, C. C., \& Wynn-Williams, C. G. 1997, ApJ, 488, 720

Elvis, M. et al. 1981, ApJ, 246, 20

Esin, A. A., et al. 2001, ApJ, 555, 483

Esin, A. A., McClintock, J. E., \& Narayan, R. 1997, ApJ, 489, 865

Esin, A.A., Narayan, R., Cui, W., Grove, J.E., et al. 1998, ApJ, 505, 854

Fabian, A.C. 2004, in "Carnegie Observatories Astrophysics Series, Vol. 1: Coevolution of Black Holes and Galaxies," ed. L. C. Ho, p447

Ferrarese, L. \& Merritt, D. 2000, ApJ, 539, L9

Fiore, F., et al. 2000, New Astronomy 5, 143

Genzel, R. et al. 1998, ApJ, 498, 579

Giacconi, R., et al. 2001, ApJ 551, 664

Griffiths, R.E., Georgantopoulos, I., Boyle, B.J., et al. 1995, MNRAS, 275, 77 
Hasinger G., Burg, R., Giacconi, R., et al. 1998, A\&A, 329, 482

Hawley, J. F. \& Balbus, S. A. 2002, ApJ, 573, 738

Ho, L.C. 1999, ApJ, 516, 672

Hornschemeier, A., et al. 2001, ApJ, 554, 742

Igumenshchev, Narayan, R., \& Abramowicz, M. A., 2003, ApJ, 592, 1042

Kato, S., Fukue, J., \& Mineshige, S. 1998, Black Hole Accretion Disks (Kyoto Univ. Press, Kyoto)

Livio, M., Pringle, J.E., \& King, A.R. 2003, ApJ, 593, 184

Magorrian, J. et al. 1998, AJ, 115, 2285

Mahadevan, R. 1999, MNRAS, 304, 501

Maiolino, R. et al. 2000, ASP Conf. Ser. 195: Imaging the Universe in Three Dimensions, 307

Maiolino, R., Comastri, A., Gilli, R., Nagar, N.M., et al. 2003, MNRAS, 344, L59

Maiolino, R., Marconi, A., Salvati, M., Risaliti, G., et al. 2001, A\&A, 365, 37

Manmoto, T., \& Kato, S. 2000, ApJ, 538, 295

Marconi, A. Moorwood, A.F.,M., Salvati, M., \& Oliva, E. 1994, A\&A, 291, 18

Marconi, A., Oliva, E., van der Werf, P.P., Maiolino, R. et al. 2000, A\&A, 357, 24

McClintock, J.E., Narayan, R., Garcia, M.R., Orosz, J.A., et al. 2003, ApJ, 593, 435

McClintock, J.E. \& Remillard, R.A., 2003, to appear in Compact Stellar X-ray Sources, eds. W.H.G. Lewin and M. van der Klis (astro-ph/0306213)

Moran, E.C., Filippenko, A.V., \& Chornock, R. 2002, ApJ, 579, L71

Mushotzky, R.F., Cowie, L.L., Barger, A.J., \& Arnaud, K.A. 2000, Nature, 404, 459

Nandra, K., et al. 1997, ApJ, 488, L91

Narayan, R., Igumenshchev, I.V., Abramowicz, M. 2000, ApJ, 539, 798

Narayan, R., Mahadevan, R., Grindlay, J.E., Popham, R.G., et al. 1998, ApJ, 492, 554 
Narayan, R., Mahadevan, R., \& Quataert, E. 1998, in "The Theory of Black Hole Accretion Discs", eds. M.A. Abramowicz, G. Bjornsson and J.E. Pringle (Cambridge University Press), p148

Narayan, R. \& Yi, I. 1995, ApJ, 444, 231

Özel, F., Psaltis, D., \& Narayan, R. 2000, ApJ, 541, 234

Page, M.J., McHardy, I.M., Gunn, K.F., Loaring, N.S., et al. 2003, AN, 324, 101

Pounds, K.A., et al. 2003, MNRAS, 341, 953

Poutanen, J., Krolik, J. H., \& Ryde, F. 1997, MNRAS, 292, L21

Ptak, A. et al. 1998, ApJ, 501, L37

Quataert, E., Di Matteo, T., Narayan, R., \& Ho, L.C. 1999, ApJ, 525, L89

Quataert, E. \& Narayan, R. 1999, ApJ, 520, 298

Quataert, E. \& Gruzinov 1999, ApJ, 520, 248

Quataert, E. \& Gruzinov 2000, ApJ, 539, 809

Rózańska, A., \& Czerny, B, 2000, A\&A, 360, 1170

Severgnini,P., Caccianiga, A., Braito, V., Della, R., et al., 2003, A\&A, 406, 483

Shields, J.C., Rix, H.-W., McIntosh, D.H., Ho, L.C et al., 2000, ApJ, 534, L27

Spoon, H.W.W., Keane, J.V., Tielens, A.G.G.M., Lutz, D., et al. 2001, A\&A, 365, L353

Spoon, H.W.W., Koornneef, J., Moorwood, A.F.M., Lutz, D., et al. 2000, A\&A, 357, 898

Stone, J., Pringle, J., \& Begelman, M. 1999, MNRAS, 310, 1002

Yuan, F., Markoff, S., \& Falcke, H., 2002a, A\&A, 383, 854

Yuan, F., Markoff, S., Falcke, H., \& Biermann, P.L. 2002b, A\&A, 391, 139

Yuan, F., Quataert, E., \& Narayan, R. 2003, ApJ, 598, 301

Zdziarski, A. A., \& Gierlinski, M. 2004, in "Stellar-mass, intermediate-mass, and supermassive black holes", Progress of Theoretical Physics, in press (astro-ph/0403683) 


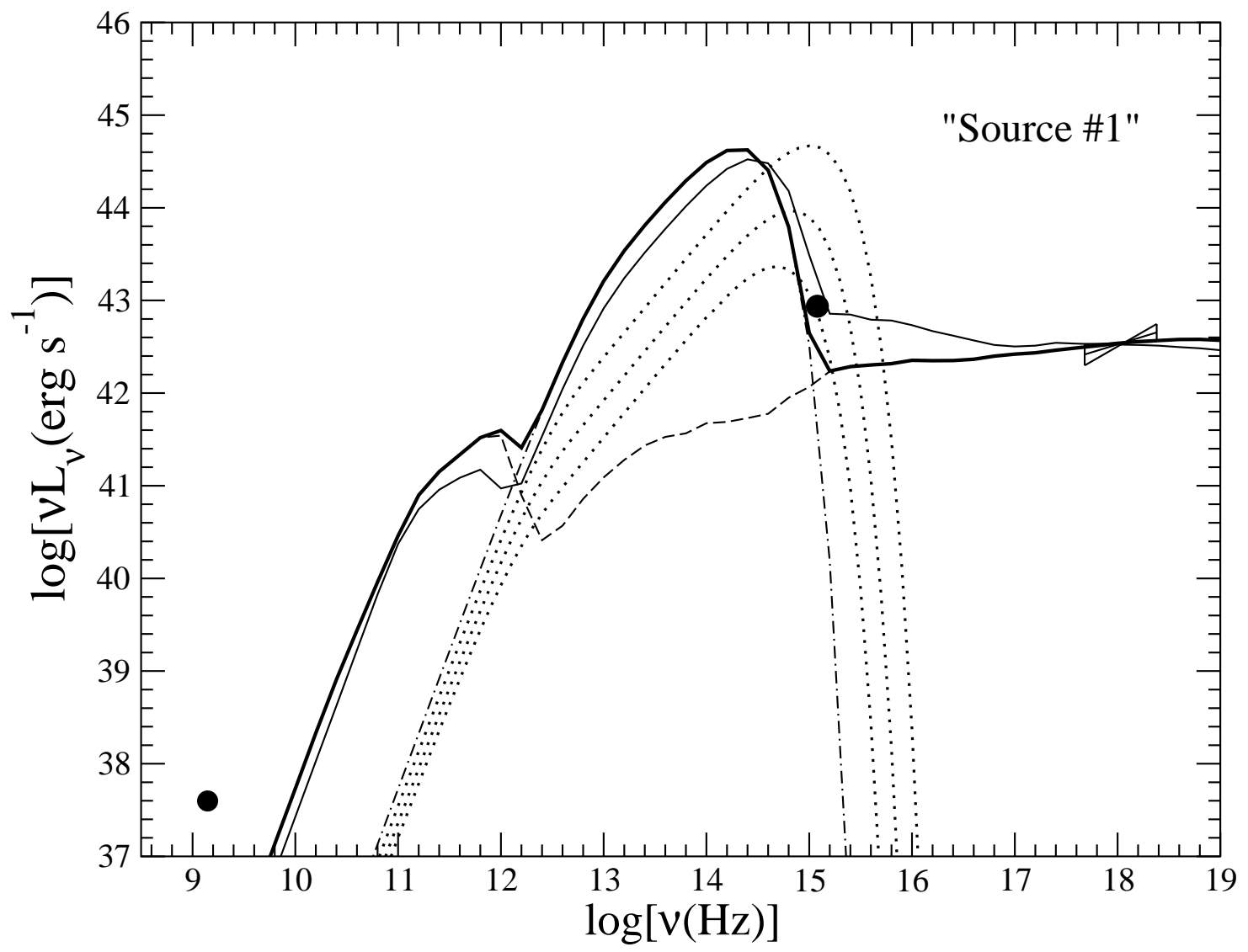

Fig. 1. - Spectral fit of the XBONG "Source \#1" (Severginini et al. 2003) with a RIAF+thin disk model. The thick solid line is the combined spectrum predicted for an accretion flow consisting of a truncated thin disk for radii $R>R_{\mathrm{tr}}=60 R_{S}$ (dot-dashed line) and a RIAF for $R<R_{\mathrm{tr}}$ (dashed line). The mass accretion rate of the RIAF at $R=R_{\mathrm{tr}}$ is $\dot{M}_{0}=10^{-2} \dot{M}_{\mathrm{Edd}}$ and it decreases with radius according to equation (1) with $s=0.3$. The thin solid line shows the result of another model (traditional ADAF) in which the accretion rate of the RIAF is taken to be independent of radius, with $\dot{M}=10^{-2} \dot{M}_{\mathrm{Edd}}$ and $R_{\mathrm{tr}}=40 R_{S}$. The three dotted lines show the emission from three standard thin accretion disks extending all the way down to $R=3 R_{S}$ with (from bottom to top) $\dot{M} / \dot{M}_{\text {Edd }}=5 \times 10^{-5}, 2 \times 10^{-4}$, and $10^{-3}$. These latter models do not fit the observations. 


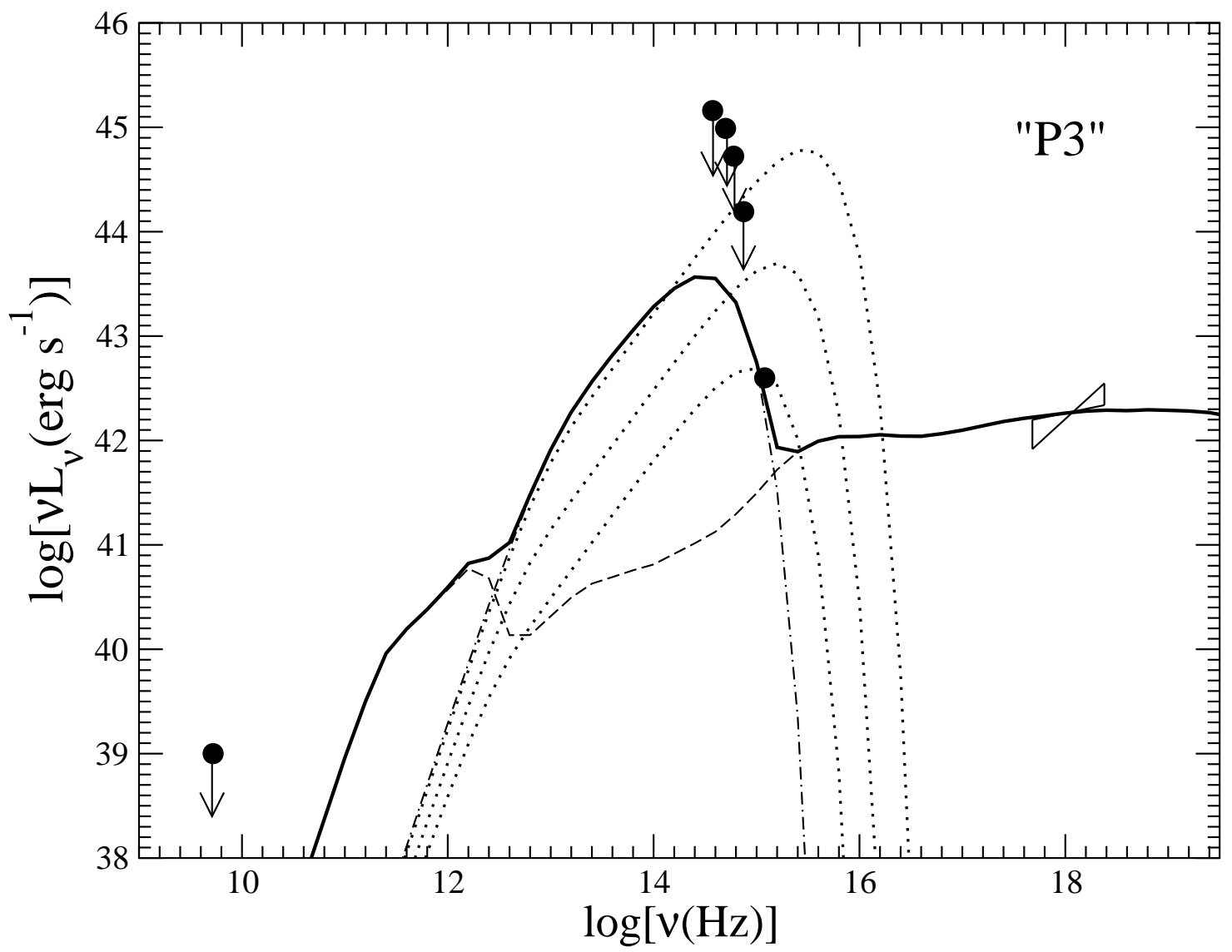

Fig. 2.- Spectral fit of the XBONG source P3 with a RIAF+thin disk model. The thick solid line is the combined spectrum predicted for an accretion flow consisting of a truncated thin disk for radii $R>R_{\mathrm{tr}}=60 R_{S}$ (dot-dashed line) and a RIAF for $R<R_{\mathrm{tr}}$ (dashed line). The mass accretion rate of the RIAF at $R=R_{\mathrm{tr}}$ is $\dot{M}_{0}=1.3 \times 10^{-2} \dot{M}_{\text {Edd }}$ and it decreases with radius according to equation (1) with $s=0.3$. The three dotted lines show the emission from three standard thin accretion disks extending all the way down to $R=3 R_{S}$ with (from bottom to top) $\dot{M} / \dot{M}_{\text {Edd }}=8 \times 10^{-5}, 8 \times 10^{-4}$, and $10^{-2}$. These latter models do not fit the observations. 


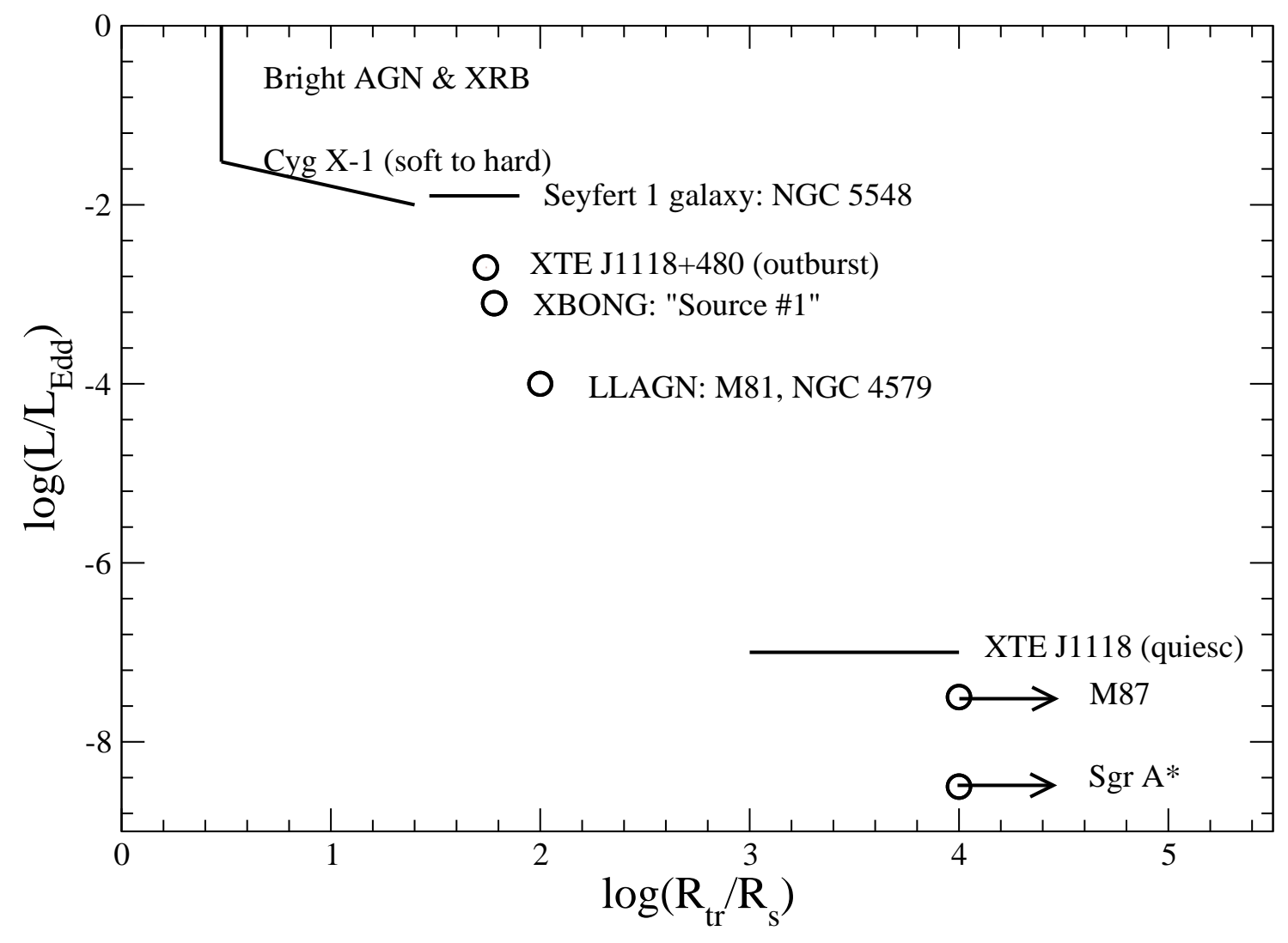

Fig. 3.- Plot of the bolometric luminosity in Eddington units along the ordinate versus the transition radius in Schwarzschild units along the abscissa for different accreting black holes. The slanting line for Cyg X-1 is based on the model of Esin et al. (1998) for the soft-to-hard state transition in this source. The other data points are from the following: Seyfert 1 galaxy NGC 5548 (Chiang \& Blaes 2003); outburst state of XTE J1118+480 (Esin et al. 2001); Source \#1 (this paper); M81 and NGC 4579 (Quataert et al. 1999); quiescent state of XTE J1118+480 (McClintock et al. 2003); M 87 (Di Matteo et al. 2003); Sgr A* (Yuan, Quataert \& Narayan 2003). 